\title{
Correction of $\mathrm{ClC}-1$ splicing eliminates chloride channelopathy and myotonia in mouse models of myotonic dystrophy
}

\author{
Thurman M. Wheeler, ${ }^{1}$ John D. Lueck, ${ }^{2}$ Maurice S. Swanson, ${ }^{3}$ \\ Robert T. Dirksen, ${ }^{2}$ and Charles A. Thornton ${ }^{1}$
}

\begin{abstract}
1Department of Neurology and 2Department of Pharmacology and Physiology, University of Rochester, Rochester, New York, USA.
${ }^{3}$ Department of Molecular Genetics and Microbiology, Genetics Institute, University of Florida, Gainesville, Florida, USA
\end{abstract}

\begin{abstract}
In myotonic dystrophy (dystrophia myotonica [DM]), an increase in the excitability of skeletal muscle leads to repetitive action potentials, stiffness, and delayed relaxation. This constellation of features, collectively known as myotonia, is associated with abnormal alternative splicing of the muscle-specific chloride channel (ClC-1) and reduced conductance of chloride ions in the sarcolemma. However, the mechanistic basis of the chloride channelopathy and its relationship to the development of myotonia are uncertain. Here we show that a morpholino antisense oligonucleotide (AON) targeting the $3^{\prime}$ splice site of $\mathrm{ClC}-1$ exon 7 a reversed the defect of ClC-1 alternative splicing in 2 mouse models of DM. By repressing the inclusion of this exon, the AON restored the full-length reading frame in ClC-1 mRNA, upregulated the level of ClC-1 mRNA, increased the expression of $\mathrm{ClC}-1$ protein in the surface membrane, normalized muscle $\mathrm{ClC}-1$ current density and deactivation kinetics, and eliminated myotonic discharges. These observations indicate that the myotonia and chloride channelopathy observed in DM both result from abnormal alternative splicing of $\mathrm{ClC}-1$ and that antisense-induced exon skipping offers a powerful method for correcting alternative splicing defects in DM.
\end{abstract}

\section{Introduction}

Dystrophia myotonica (myotonic dystrophy) type 1 (DM1), the most common muscular dystrophy affecting adults, is caused by expansion of a CTG repeat in the 3' untranslated region of the gene encoding the DM protein kinase (DMPK) (1). Evidence suggests that DM1 is not caused by abnormal expression of DMPK protein, but rather that it involves a toxic gain of function by mutant $D M P K$ transcripts that contain an expanded CUG repeat (CUG ${ }^{\exp }$ ) (reviewed in ref. 2). The transcripts containing a CUG $G^{\exp }$ tract elicit abnormal regulation of alternative splicing, or spliceopathy (3). The splicing defect, which selectively affects a specific group of pre-mRNAs, is thought to result from reduced activity of splicing factors in the muscleblind (MBNL) family (4), increased levels of CUG-binding protein $1(3,5)$, or both. Decreased activity of MBNL proteins can be attributed to sequestration of these proteins in nuclear foci of CUG ${ }^{\exp } \mathrm{RNA}(6,7)$.

Previously we showed that transgenic mice expressing CUGexp RNA (human skeletal actin long repeat $\left[H S A^{L R}\right]$ mice) displayed myotonia and chloride channel 1 (ClC-1) splicing defects similar to those observed in DM1 (8). We postulate that myotonia in the $H S A^{L R}$ model results from abnormal inclusion of exon $7 \mathrm{a}$ in the ClC-1 mRNA, owing to sequestration of MBNL1, a factor required for repression of exon 7a splicing in muscle fibers (4). This mechanism is supported by several lines of evidence: (a) inclusion of exon 7 a causes frame shift and introduction of a premature termination codon in the ClC-1 mRNA $(5,8)$; (b) trun-

Nonstandard abbreviations used: $\mathrm{AON}$, antisense oligonucleotide; $\mathrm{ClC}-1$, chloride channel 1; CUG ${ }^{\exp }$, expanded CUG repeat; DM, dystrophia myotonica (myotonic dystrophy); DMPK, DM protein kinase; EMG, electromyography; FDB, flexor digitorum brevis; $H S A^{L R}$, human skeletal actin long repeat; MBNL, muscleblind; Po, open probability; TA, tibialis anterior.

Conflict of interest: The authors have declared that no conflict of interest exists. Citation for this article: J. Clin. Invest. 117:3952-3957 (2007). doi:10.1172/JCI33355 cated ClC-1 protein encoded by the exon $7 \mathrm{a}+$ isoform is devoid of channel activity (9); and (c) disruption of Mbnl1 in mice leads to increased inclusion of $\mathrm{ClC}-1$ exon $7 \mathrm{a}$ and myotonia (4). Our postulate that myotonia in DM1 results from deficiency of ClC-1 is based on observations that mouse models of DM1 display a $70 \%-80 \%$ reduction in muscle chloride conductance $(8,10)$, coupled with previous estimates that a $75 \%$ reduction of $\mathrm{ClC}-1$ conductance is sufficient to cause myotonic discharges in muscle fibers (11). However, the mechanism of ClC-1 downregulation and its requirement for myotonia in DM1 are controversial. Effects on sodium or potassium channels have also been implicated in DM1-associated myotonia (12-14). In addition, evidence that chloride channelopathy in DM1 results from downregulation of ClC-1 transcription, rather than abnormal splicing, has been reported (15). To provide a causal link between ClC-1 alternative splicing, chloride channelopathy, and myotonia in DM1, we used a morpholino antisense oligonucleotide (AON) to selectively repress the inclusion of exon $7 \mathrm{a}$.

\section{Results}

The strategy for suppressing the inclusion of exon $7 \mathrm{a}$ is diagrammed in Figure 1A. The morpholino AONs were complementary to the $3^{\prime}$ or $5^{\prime}$ splice sites of exon $7 \mathrm{a}$ in the ClC-1 pre-mRNA (Figure 1B). To examine tissue uptake, we injected a carboxyfluorescein-labeled morpholino into tibialis anterior (TA) muscle of $H S A^{L R}$ mice. Examination of tissue sections indicated that uptake of antisense morpholino was limited to the needle track (data not shown). To improve uptake and distribution of AON, we used voltage pulses to electroporate muscle fibers after the AON injection. This led to uptake of antisense morpholino throughout the TA muscle (Figure 2, A-C). Of note, the AON was present in both nucleus and cytoplasm but appeared to accumulate preferentially in the nucleus (Figure 2, A and E). 
A

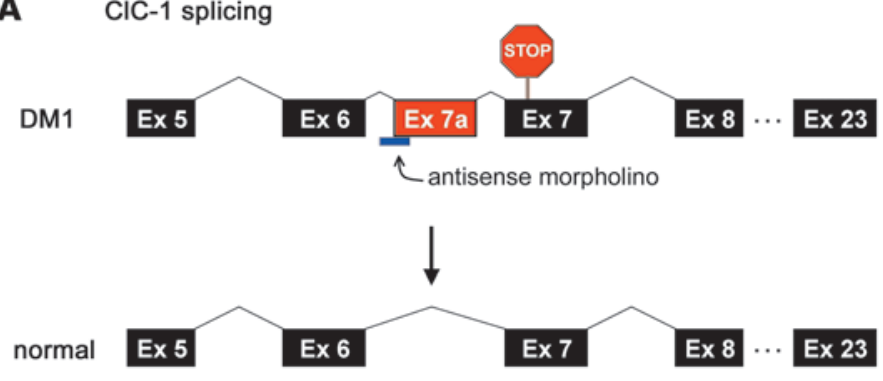

B

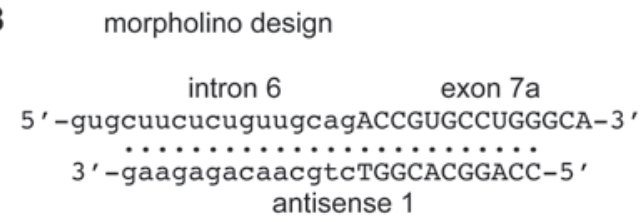
exon $7 a$
exon 7a intron 7a
$5^{\prime}-$ GCCCCTGAUGGAGgcaaguuucacuuccucc-3'
$\ldots \ldots \ldots \ldots \ldots \ldots \ldots$
3'-GGACTACCTC. antisense 2

\section{$5^{\prime}$-gaagagacaacgtcTGGCACGGACC-3' invert control}

\section{Figure 1}

Design of antisense morpholinos. (A) Inclusion of CIC-1 exon 7a induces a frame shift and premature termination codon in exon 7. Annealing of antisense morpholino to the $3^{\prime}$ splice site of exon $7 \mathrm{a}$ in the CIC-1 pre-mRNA is intended to prevent spliceosomal recognition of this exon. (B) Alignment of CIC-1 pre-mRNA (top strand) with antisense morpholinos targeting the $3^{\prime}$ or $5^{\prime}$ splice sites of exon 7 a is shown. The control morpholino is the $5^{\prime}-3^{\prime}$ invert of the $3^{\prime}$ splice site blocker. Exonic sequences are in upper case, intronic sequences are in lower case.

To determine the effect of morpholino on splicing in $H S A^{L R}$ mice, total RNA was extracted from TA muscle after AON injection. Analysis of ClC- 1 splicing by RT-PCR showed that antisense morpholino had the intended effect of suppressing the inclusion of exon $7 \mathrm{a}$, whereas control morpholino with inverted sequence had no effect on ClC-1 splicing in the contralateral TA (Figure 3, $A$ and D). AON targeting the $3^{\prime}$ splice site, or coinjection of AONs targeting the $3^{\prime}$ and $5^{\prime}$ splice sites, was more effective than targeting the $5^{\prime}$ splice site alone (Supplemental Figure 1; supplemental material available online with this article; doi:10.1172/ JCI33355DS1). Effective and sustained skipping of exon 7a was achieved after a single injection of morpholino AON. Inclusion of exon 7a was suppressed to WT levels for at least 3 weeks after a single injection (Figure 3, A and D), and a partial exclusion of exon 7 a was still evident after 8 weeks (Figure 3, B and E). Notably, the antisense morpholino did not affect the formation of nuclear foci containing CUG ${ }^{\exp }$ RNA and MBNL1 protein (Supplemental Figure 2), nor did it correct the alternative splicing of other genes that are misregulated in DM1, such as titin (Figure 3C), ZASP, or Serca1 (data not shown) (7). These data indicate that morpholino AON specifically corrects the $\mathrm{ClC}-1$ splicing defect rather than producing a general reversal of DM-associated spliceopathy or Mbnl1 sequestration.

Morpholino AONs influence splicing outcomes without inducing degradation of their target RNAs (16). Therefore, the predicted effect of repressing exon $7 \mathrm{a}$ inclusion was to eliminate the premature termination codon in ClC- 1 mRNA and thereby reduce its degradation through the nonsense-mediated decay pathway (17). Consistent with this prediction, treatment with antisense morpholino, but not control morpholino with inverted sequence, led to increased levels of ClC-1 mRNA, as determined by quantitative realtime RT-PCR (Figure 3F). These results indicate
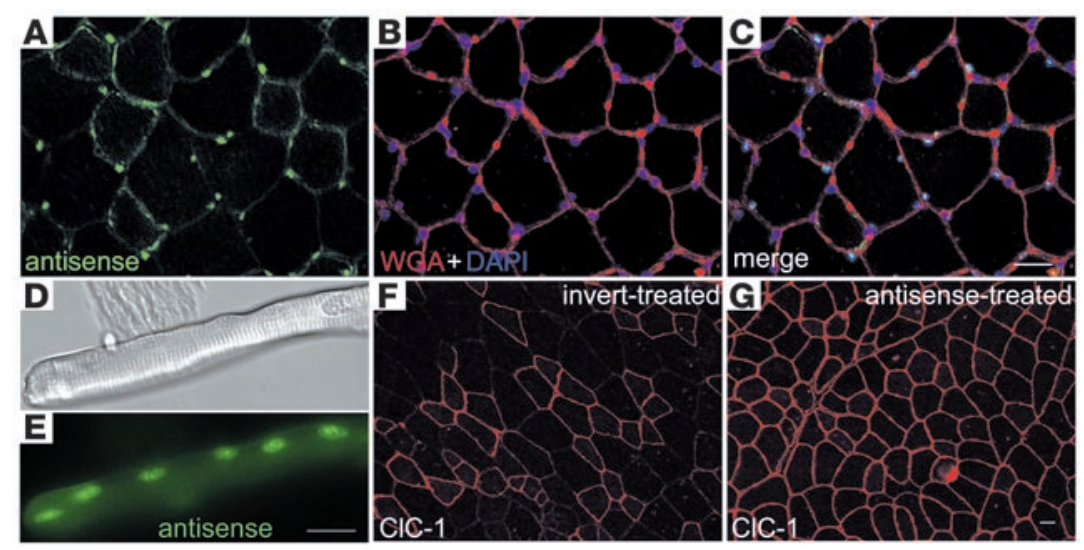

\section{Figure 2}

Antisense morpholino localizes preferentially to muscle nuclei and restores $\mathrm{CIC}-1$ expression at the sarcolemma. (A-C) Cross section of HSALR TA muscle showing distribution of $3^{\prime}$-carboxyfluorescein-labeled antisense morpholino (green) 3 weeks after injection. The morpholino was complementary to the $3^{\prime}$ splice site of CIC-1 pre-mRNA. Muscle fibers are outlined by wheat germ agglutinin (WGA; red), and nuclei are highlighted by DAPI (blue). Bright field (D) and fluorescence (E) images of a single FDB fiber showing preferential nuclear localization of the antisense morpholino (day 5 after injection). As compared with invert-treated control (F), immunofluorescence shows an increase of sarcolemmal CIC-1 protein (red) in HSA LR TA muscle 3 weeks after treatment with antisense morpholino (G). Scale bars: $20 \mu \mathrm{m}$. 


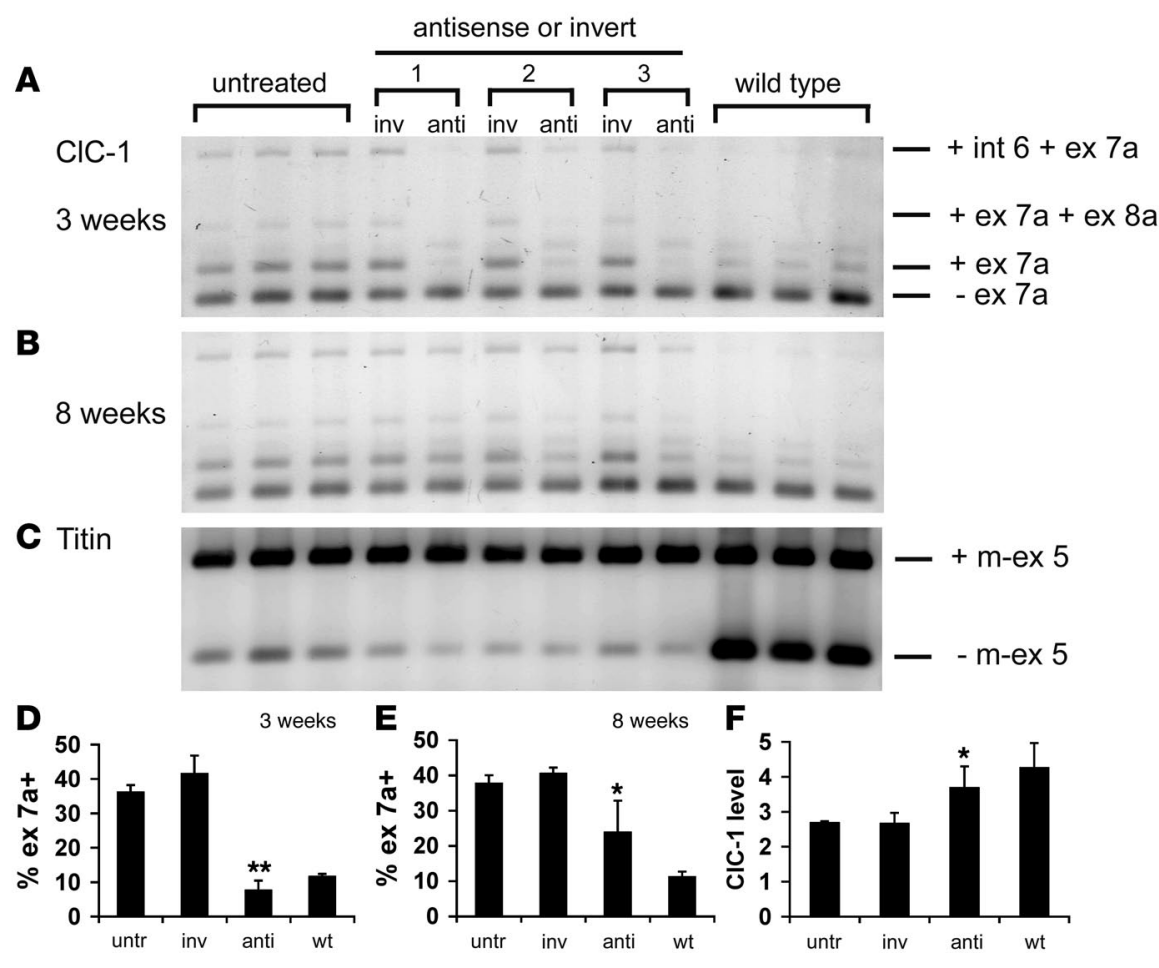

Figure 3

Antisense morpholino represses splicing of CIC-1 exon 7a. (A) RT-PCR showed reduction of exon 7a inclusion 3 weeks after injection of antisense (anti) morpholino (antisense $1+$ antisense 2; $5 \mu \mathrm{g}$ each) into TA muscle of $H S A^{L R}$ mice. Pairs of injected TA muscles from each mouse are identified as 1,2, and 3. Muscle injected with control morpholino (inv) $(10 \mu \mathrm{g})$ was not different from untreated $H S A^{L R}$ muscle. $H S A^{L R}$ and WT mice have the same (FVB) inbred strain background. int, intron, ex, exon. (B) Inclusion of exon 7a remained partially suppressed 8 weeks after injection of antisense morpholino (20 $\mu \mathrm{g}$ antisense $1 \mathrm{vs} .20 \mu \mathrm{g}$ invert control). (C) CIC-1 antisense morpholino did not correct the misregulated alternative splicing of titin M-line exon 5 . The percentage of $\mathrm{ClC}-1$ splice products that include exon $7 \mathrm{a}$ is shown at 3 (D) and 8 (E) weeks following morpholino injection. Mean $\pm \mathrm{SD} ; n=3$ per group; ${ }^{* *} P<0.001 ;{ }^{*} P=0.035$ antisense versus invert-treated controls; $t$ test. (F) The level of CIC-1 mRNA was increased 3 weeks after treatment with antisense moropholino. CIC-1 mRNA level is expressed in arbitrary units relative to housekeeping gene RNA polymerase II transcription factor IIB. Mean \pm SD; $n=3$ per group; ${ }^{\star} P=0.06$ for antisense versus invert-treated control; $t$ test. is sufficient to completely restore normal ClC-1 current density and channel deactivation kinetics.

Next we determined the effects of repressing exon 7 a inclusion on muscle physiology in vivo. Electromyography (EMG) analysis by an examiner blinded to the experimental protocol revealed that myotonia was markedly reduced or absent in TA muscles of $H S A^{L R}$ mice after injection of antisense morpholino, whereas myotonia in the invertinjected contralateral TA was not different from that in uninjected muscle (Figure 4, E and F). Myotonia reduction correlated with the degree of exon 7a skipping at 3- and 8week time points, indicating that a single injection of antisense morpholino provided a sustained reduction in myotonia.

Previously we showed that homozygous deletion of Mbnl1 exon 3 in mice $\left(M b n l 1^{\triangle E 3 / \triangle E 3}\right)$ resulted in loss of Mbnl1 protein from muscle, spliceopathy similar to that in DM1 patients and $H S A^{L R}$ mice, reduction of $\mathrm{ClC}-1$ expression and activity, and myotonia $(4,7,10)$. To examine the effects of AON in this model, we injected antisense morpholino or invert control into TA muscle of Mbnl1 ${ }^{\triangle E 3 / \triangle E 3}$ mice. As in the $H S A^{L R}$ transgenic model, antisense morpholino repressed the inclusion of exon 7a (Figure 5, A and B), increased the expression of $\mathrm{ClC}-1$ protein at the sarcolemma (Figure 5, C and D), and reduced the myotonia in $M b n l 1^{\triangle E 3 / \Delta E 3}$ mice (Figure 5E). Thus, while the pathogenesis of spliceopathy in DM is a subject of debate, rescue of the myotonia by antisense morpholino does not depend on the exact manner in which the ClC-1 splicing defect is generated. labeled antisense or invert morpholino. Patch-clamp analysis was performed 3-5 days after injection, at a time when fibers were still small enough to maintain an effective voltage clamp (10). Individual FDB fibers were isolated, and macroscopic $\mathrm{ClC}-1$ channel activity was measured in fibers exhibiting green fluorescence (Figure 2, D and E). ClC-1 current density (Figure 4, A and B) and deactivation kinetics (Figure 4D) were rescued to WT values as early as 3 days after morpholino AON injection, while current density and deactivation kinetics in fibers treated with invert morpholino were not different from those of untreated $H S A^{L R}$ fibers. The slower rate of channel deactivation observed for WT and AON-treated fibers was most likely not due to a current-dependent effect on channel gating, as reducing WT $\mathrm{ClC}-1$ current magnitude in half with a prepulse does not significantly alter the kinetics of channel deactivation (10). Rescue of ClC-1 activity was not due to a shift in channel activation, since the voltage dependence of relative channel open probability $(\mathrm{Po})$ was not different between antisense- and invert-injected $H S A^{L R}$ and WT fibers (Figure 4C). These results demonstrate that morpholino $\mathrm{AON}$ rescue of $\mathrm{ClC}-1$ spliceopathy

\section{Discussion}

Current models of DM1 pathogenesis postulate that DMPK mRNA containing a CUG ${ }^{\exp }$ alters the function of splicing factors, leading to misregulated alternative splicing for a specific group of pre-mRNAs (3). In operational terms, one difficulty with this model is that functional differences between alternative splice isoforms, or biological consequences of altering the ratio of 2 alternative splice products, are not easy to determine. Furthermore, in the context of dozens to hundreds of transcripts whose splicing is so affected, the phenotypic consequences of any particular splicing change may be difficult to ascertain. A key finding of the present study is that misregulated alternative splicing of ClC- 1 is required for the development of myotonia, a cardinal symptom of DM1, in both $H S A^{L R}$ and $M b n l 1^{\triangle E 3 / \triangle E 3}$ mice. To our knowledge, these results provide the clearest indication to date that $\mathrm{CUG}^{\mathrm{exp}}$-induced spliceopathy is directly involved in producing a clinical feature of DM1. Furthermore, our results suggest an approach for dissecting the functional significance of any particular splicing change. AONs can be used to correct a specific splicing defect in DM1 cells or to induce a DM1-like effect in WT cells. 
A
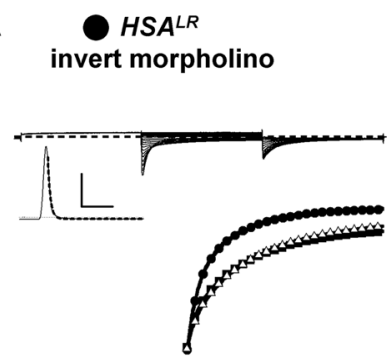

B
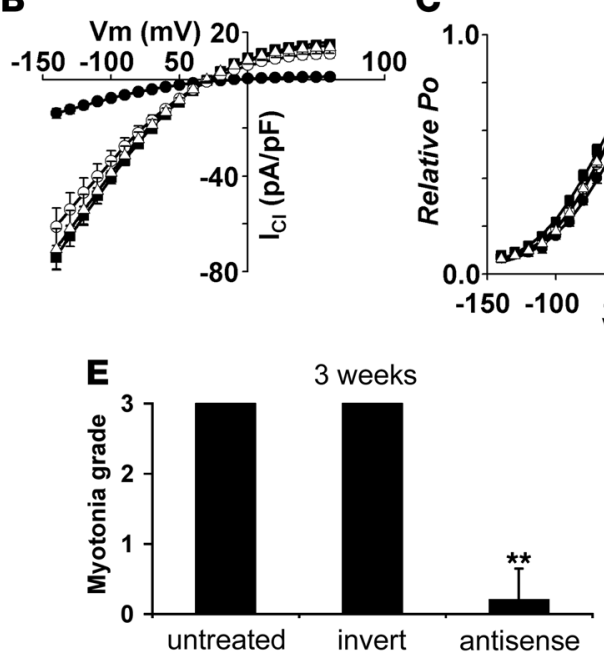

HSALR antisense morpholino

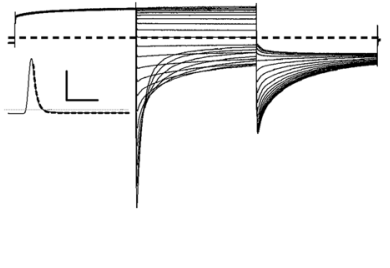

C

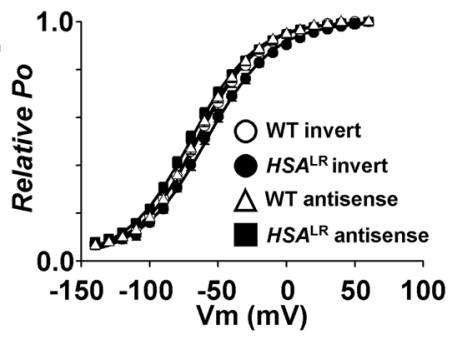

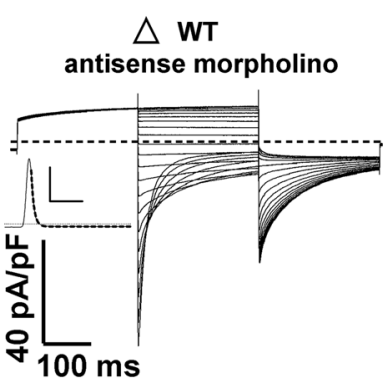

D

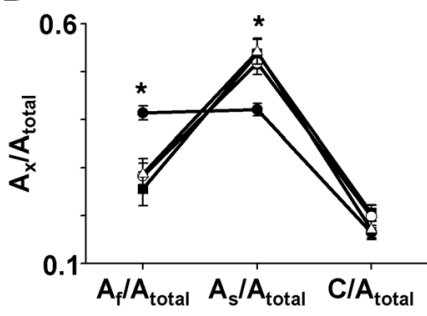

$\mathbf{F}$

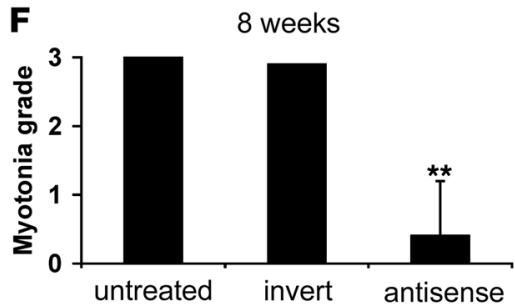

Figure 4

Antisense morpholino rescues CIC-1 channel function and reverses myotonia in skeletal muscle of $H S A^{L R}$ mice. (A) Representative CIC-1 currents obtained from FDB fibers isolated from $H S A^{L R}$ mice electroporated with either invert (left) or antisense (middle) morpholino and WT mice electroporated with antisense morpholino (right). The dashed lines represent the 0 current level. Capacitative currents recorded from each fiber are shown in the inset of each panel (scale bars: vertical, $3 \mathrm{nA}$; horizontal, $4 \mathrm{~ms}$ ). Superimposed traces (solid lines) of normalized CIC-1 current deactivation at $-100 \mathrm{mV}$ in FDB fibers obtained from invert- (circles) and antisense-treated (squares) $H S A^{L R}$ mice and antisense-treated WT mice (triangles) fit with a second-order exponential are shown in the inset to the left panel. Note that accelerated CIC-1 deactivation kinetics of FDB fibers obtained from $H S A^{L R}$ mice are normalized only following treatment with antisense morpholino. (B) Membrane potential (Vm) dependence of average instantaneous CIC-1 current density recorded from FDB fibers of 16- to 18-day-old WT mice treated with invert morpholino $(n=11)$, WT mice treated with antisense morpholino $(n=10), H S A^{L R}$ mice treated with invert morpholino $(n=12)$, and $H S A^{L R}$ mice treated with antisense morpholino $(n=16)$. (C) Average relative Po-Vm curves for the same experiments shown in $\mathbf{B}$. Smooth curves through each data set were generated using a modified Boltzmann equation (10). (D) Average relative contribution of the fast $\left(A_{f} / A_{\text {total }}\right)$, slow $\left(A_{s} / A_{\text {total }}\right)$, and nondeactivating $\left(\mathrm{C} / \mathrm{A}_{\text {total }}\right)$ components of $\mathrm{CIC}-1$ current deactivation elicited from a voltage step to $-100 \mathrm{mV}$ for the same experiments shown in $\mathrm{B}$. Mean $\pm \mathrm{SEM}$; ${ }^{\star} P<0.05$ invert-treated $H S A^{L R}$ fibers compared with each of the other experimental conditions; $t$ test. Myotonia was significantly reduced 3 (E) and $8(F)$ weeks following injection of antisense morpholino. Mean \pm SD; $n=3-7$ per group. Antisense morpholino was injected into one TA; invert morpholino was injected into the contralateral TA; and gastrocnemius muscle served as an untreated control. ${ }^{\star \star} P<0.0001$ for antisense versus invert-treated control; ANOVA.

Myotonia is the symptom by which DM1 is most often recognized, and, due to preferential involvement of hand and forearm muscles, it compromises manual dexterity and contributes to disability. In light of the abnormal calcium homeostasis observed in DM1 cells and model systems $(18,19)$, and the effects of DM1 on alternative splicing of the SERCA1 calcium reuptake pump of the sarcoplasmic reticulum $(7,20)$, it also seems possible that excessive calcium release due to myotonic discharges may aggravate the degeneration of DM1 muscle fibers. Although previous studies have implicated sodium channels or calcium-activated potassium channels in DM1 (12-14), a second finding of the present study is that DM1-associated myotonia results primarily from a chloride channelopathy.

We previously found that the number of functional ClC- 1 channels in the sarcolemma was markedly decreased, the rate of channel deactivation was increased, and the maximum ClC-1 channel
Po was reduced in both $H S A^{L R}$ and $M b n l 1^{1 E 3 / \triangle E 3}$ mice (10). The observed acceleration in channel deactivation and reduction in maximal channel Po are consistent with previously reported dominant-negative effects imparted by exon 7a-encoded protein products (9). Our observations that electroporation of AON in $H S A^{L R}$ muscle reduced levels of exon 7a-containing transcript (Figure 3, $\mathrm{D}$ and $\mathrm{E}$ ), increased full-length ClC-1 transcript (Figure $3 \mathrm{~F}$ ), and completely normalized ClC-1 current density (Figure 4B) and deactivation gating (Figure 4D) support the assertion that chloride channelopathy in DM1 involves a complex combination of transdominant RNA- and protein-based mechanisms.

AONs influence RNA processing by annealing to pre-mRNA and blocking the access of splicing factors to splice sites or cis-acting regulatory elements (21). AONs that induce skipping of constitutively spliced exons have been used to bypass stop codons or 
A

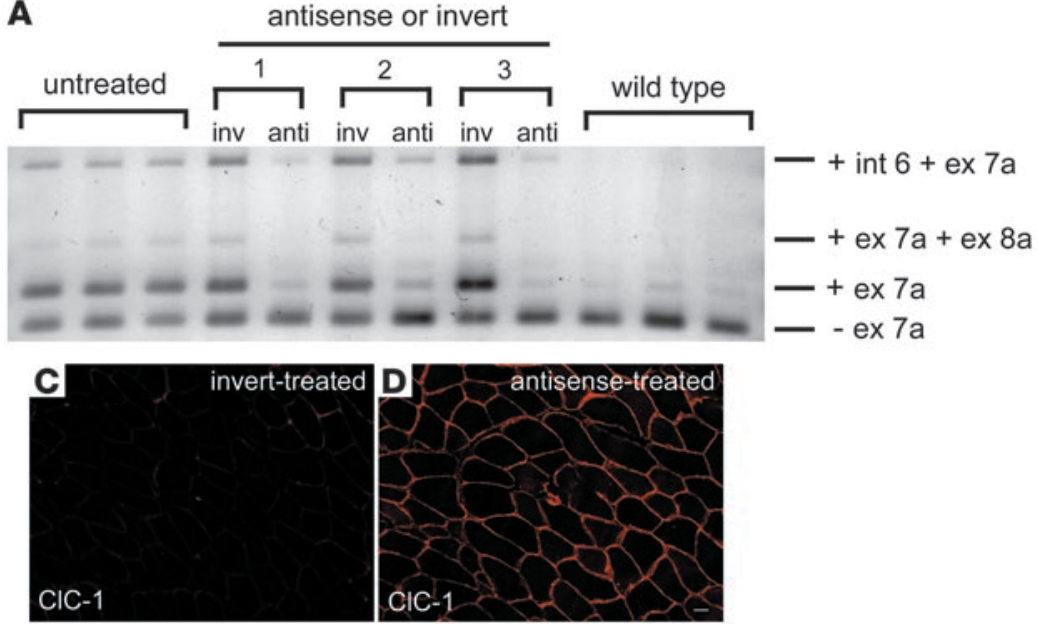

B
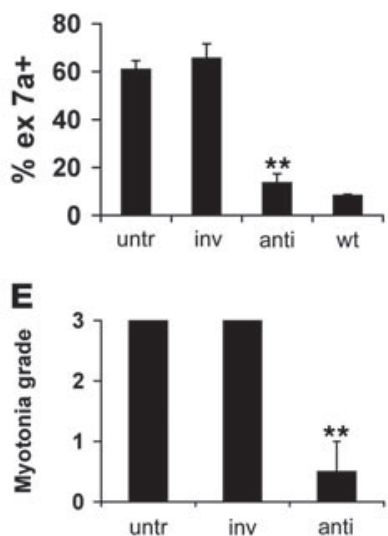

\section{Figure 5}

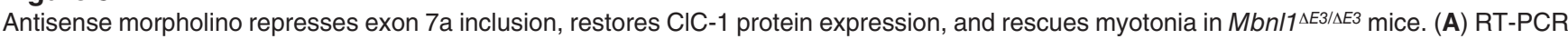
shows reduced inclusion of exon 7a at 3 weeks after injection of antisense morpholino 1 (20 $\mu \mathrm{g}$ antisense or invert control). (B) Quantitation of splicing results shown in $\mathbf{A}$ as mean $\pm \mathrm{SD} ; n=3$ per group; ${ }^{* *} P<0.001$, antisense versus invert-treated control; $t$ test. Immmunofluorescence for CIC-1 is increased 3 weeks after injection with antisense (D) as compared with invert-treated control (C). Scale bar: $20 \mu \mathrm{m}$. (E) Myotonia in Mbn/1 ${ }^{\triangle E 3 / \triangle E 3}$ TA muscle was reduced 3 weeks after treatment with antisense morpholino but not in muscle treated with invert control. Mean \pm $\mathrm{SD} ; n=3$ per group; ${ }^{* *} P<0.0001$, antisense versus invert-treated control; ANOVA.

restore the proper reading frame in the dystrophin mRNA (22-24). We postulated that exon 7 a may show heightened susceptibility to AONs because splicing signals in alternative exons tend to be intrinsically weak and this exon is normally skipped in a fraction of ClC-1 transcripts (17). However, ClC-1 mRNAs that include exon 7 a contain premature termination codons and undergo rapid degradation (17). Therefore, these splice products are underrepresented at steady state, and we cannot determine the exact efficiency of AON-induced exon skipping. Despite this limitation, the decrease of exon $7 \mathrm{a}+$ isoforms to WT levels and the normalized activity of $\mathrm{ClC}-1$ channels in treated muscle fibers suggest that this intervention is highly effective and surprisingly prolonged.

To our knowledge, these results are the first to show that symptoms of DM1 are reversible using a targeted approach to restore normal alternative splicing that does not rely on gene therapy. While several drugs with antimyotonia properties are currently available, they provide only partial relief of symptoms, and their use in DM1 is limited by the lack of controlled trials supporting their efficacy and safety (25). Results here indicate that targeting the $\mathrm{ClC}-1$ splicing defect could be highly effective for treating the myotonia in DM1.

\section{Methods}

Design of oligonucleotides. Morpholino oligonucleotides (Gene Tools LLC) were 5'-CCAGGCACGGTctgcaacagagaag-3', targeting the ClC-1 3' splice site; $5^{\prime}$-gaagagacaacgtctggcacggacc- $3^{\prime}$ inverted control; and $5^{\prime}$-ggaagtgaacttgcCTCCATCAGG-3', targeting the ClC-1 $5^{\prime}$ splice site. (Upper case indicates exonic sequences, lower case indicates intronic sequences.)

Morpholino injections. Animal experiments were approved by the University Committee on Animal Resources at the University of Rochester Medical Center. $H S A^{L R}(26)$ or $M b n l 1^{\triangle E 3 / \triangle E 3}$ mice (4) were anesthetized by intraperitoneal injection of $100 \mathrm{mg} / \mathrm{kg}$ ketamine, $10 \mathrm{mg} / \mathrm{kg}$ xylazine, and $3 \mathrm{mg} / \mathrm{kg}$ acepromazine. TA muscle was pretreated by intramuscular injection of bovine hyaluronidase (15 $\mu \mathrm{l}, 0.4 \mathrm{U} / \mu \mathrm{l})$ (Sigma-Aldrich) (27). Two hours later, 10 or $20 \mu \mathrm{g}$ of morpholino in a total volume of $20 \mu \mathrm{l}$ PBS was injected using a 30-gauge needle. TA muscle was then electroporated using electrodes placed parallel to the long axis of the muscle. Electroporation parameters were $100 \mathrm{~V} / \mathrm{cm}, 10$ pulses at $1 \mathrm{~Hz}$, and 20-ms duration per pulse. Antisense or control morpholino with inverted sequence was injected into TA muscles of opposite limbs. The determination of which TA received antisense morpholino was randomized, and investigators remained blinded to this assignment until EMG analyses were completed. Other analyses were performed without blinding. For experiments to determine the distribution of injected oligonucleotides, the antisense morpholino was labeled with carboxyfluorescein, and cryosections of muscle $(10 \mu \mathrm{M})$ were examined by fluorescence microscopy, with or without fixation in $4 \%$ paraformaldehyde. Some sections were colabeled with TRITC-wheat germ agglutinin (28) $(50 \mu \mathrm{g} / \mathrm{ml}$ in PBS; Sigma-Aldrich) and DAPI to highlight the surface membranes and nuclei of muscle fibers.

$R N A$ analysis. Mice were sacrificed 3 or 8 weeks after morpholino injection. TA muscles were removed and frozen in liquid nitrogen. Total RNA was isolated with TriReagent (Molecular Research Center). cDNA synthesis was primed with oligo-dT as described previously (8). Assays for alternative splicing of $\mathrm{ClC}-1$ and titin were described previously $(7,8)$. Primer sequences were: ClC-1 forward, ClCm-7 5'-TGAAGGAATACCTCACACTCAAGG3', and reverse, ClCm-30 5'-CACGGAACACAAAGGCACTG-3'; mtitin forward, mTTN1 5'-GTGTGAGTCGCTCCAGAAACG-3', and reverse, mTTN2 5'-CCACCACAGGACCATGTTATTTC-3'.

RT-PCR products (22 cycles) were separated on agarose gels, stained with SYBR green II, and scanned on a laser fluorimager (Molecular Dynamics). Band intensity was quantified using ImageQuant software (GE Healthcare). Total levels of ClC-1 mRNA were determined by quantitative realtime RT-PCR (Taqman; Applied Biosystems) relative to housekeeping gene RNA polymerase II transcription factor IIB.

Immunofluorescence. Frozen transverse sections of TA muscle $(10 \mu \mathrm{M})$ were stained with affinity-purified rabbit polyclonal anti-ClC-1 antibody (1:50; Alpha Diagnostic International) as previously described (29). Muscle sections from ClC-1-null mice and WT FVB mice served as negative and positive controls on each slide (data not shown). $z$-Plane stacks consisting 
of 8 images separated by $0.25 \mu \mathrm{M}$ were captured and deconvolved using Autoquant version 9.3 software (Autoquant Imaging). Maximum projection images were obtained using Metavue software (Universal Imaging Corp.). Exposure time and thresholding were identical for all comparisons of antisense and invert controls.

Macroscopic recordings of ClC-1 current. Delivery of antisense and invert morpholinos into FDB fibers was achieved by injection and electroporation of hind limb footpads. Briefly, 12- to 14-day-old $H S A^{L R}$ mice were anesthetized by intraperitoneal injection of $100 \mathrm{mg} / \mathrm{kg}$ ketamine, $10 \mathrm{mg} / \mathrm{kg}$ xylazine, and $3 \mathrm{mg} / \mathrm{kg}$ acepromazine. Hind limb foot pads then were injected with bovine hyaluronidase followed 1 hour later with $20 \mu \mathrm{g}(10 \mu \mathrm{l}, 2 \mathrm{mg} / \mathrm{ml}$ in PBS) of antisense or invert carboxyfluorescein-labeled morpholino. Uptake of morpholinos was enhanced by electroporation $(100 \mathrm{~V} / \mathrm{cm}$, 20 pulses at $1 \mathrm{~Hz}$, and $20 \mathrm{~ms}$ per pulse) of the foot pad immediately after injection (30). Three to 5 days after injection/electroporation, individual FDB muscle fibers were isolated as previously described (10). Bright field and fluorescence (488 $\mathrm{nm}$ excitation) images of single FDB fibers were acquired using a 40x (1.4 NA) objective and a TILL IMAGO-QE cooledCCD camera. Only fibers exhibiting clear striations, clean surfaces, and green fluorescence were chosen for electrophysiological recordings. ClC-1 currents were measured and analyzed in whole-cell patch clamp experiments (31) using an approach identical to that described in detail elsewhere (10). ClC-1 current density $(\mathrm{pA} / \mathrm{pF})$ was calculated in order to compare data across fibers of different sizes.

$E M G$. EMG was performed under general anesthesia as described previously (4). Images and video recordings of electromyographic myotonia in $H_{S A} A^{L R}$ and $M b n l 1^{\triangle E 3 / \triangle E 3}$ mice are shown in previous reports $(4,26)$. A minimum of 15 needle insertions were performed for each muscle examined. Myotonic discharges were graded on a 4-point scale: 0 , no myotonia; 1 , occasional myotonic discharge in less than $50 \%$ of needle insertions; 2 , myotonic discharge in greater than $50 \%$ of needle insertions; 3 : myotonic discharge with nearly every insertion.

Statistics. Group data are expressed as mean \pm SD, except for patch clamp data in Figure 4, which are expressed as mean \pm SEM. Between-group comparison was performed by 2-tailed Student's $t$ test or 1-way ANOVA as indicated. A $P$ value $<0.05$ was considered significant.

\section{Acknowledgments}

The authors thank Matt Krym, Linda Richardson, and Kirti Bhatt for technical assistance. This work was supported by the University of Rochester Senator Paul D. Wellstone Muscular Dystrophy Cooperative Research Center (NIH/NS48843), with additional support from the NIH (AR46806, AR/NS48143), the Run America Foundation, the Saunders Family Neuromuscular Research Fund, the Schwab Research Fund, and a National Institute of Dental and Craniofacial Research training grant (T32DE07202).

Received for publication July 23, 2007, and accepted in revised form September 12, 2007.

Address correspondence to: Charles Thornton, Box 673, University of Rochester Medical Center, 601 Elmwood Avenue, Rochester, New York 14618, USA. Phone: (585) 275-2542; Fax: (585) 2731255; E-mail: charles_thornton@urmc.rochester.edu.
1. Brook, J.D., et al. 1992. Molecular basis of myotonic dystrophy: expansion of a trinucleotide (CTG) repeat at the $3^{\prime}$ end of a transcript encoding a protein kinase family member. Cell. 68:799-808.

2. Osborne, R.J., and Thornton, C.A. 2006. RNA-dominant diseases. Hum. Mol. Genet. 15:R162-R169.

3. Philips, A.V., Timchenko, L.T., and Cooper, T.A. 1998. Disruption of splicing regulated by a CUGbinding protein in myotonic dystrophy. Science. 280:737-741.

4. Kanadia, R.N., et al. 2003. A muscleblind knockout model for myotonic dystrophy. Science. 302:1978-1980.

5. Charlet, B.N., et al. 2002. Loss of the muscle-specific chloride channel in type 1 myotonic dystrophy due to misregulated alternative splicing. Mol. Cell. 10:45-53.

6. Miller, J.W., et al. 2000. Recruitment of human muscleblind proteins to (CUG)(n) expansions associated with myotonic dystrophy. EMBO J. 19:4439-4448.

7. Lin, X., et al. 2006. Failure of MBNL1-dependent postnatal splicing transitions in myotonic dystrophy. Hum. Mol. Genet. 15:2087-2097.

8. Mankodi, A., et al. 2002. Expanded CUG repeats trigger aberrant splicing of $\mathrm{ClC}-1$ chloride channel pre-mRNA and hyperexcitability of skeletal muscle in myotonic dystrophy. Mol. Cell. 10:35-44.

9. Berg, J., Jiang, H., Thornton, C.A., and Cannon, S.C. 2004. Truncated ClC-1 mRNA in myotonic dystrophy exerts a dominant-negative effect on the Cl current. Neurology. 63:2371-2375.

10. Lueck, J.D., Mankodi, A., Swanson, M.S., Thornton, C.A., and Dirksen, R.T. 2007. Muscle chloride channel dysfunction in two mouse models of myotonic dystrophy. J. Gen. Physiol. 129:79-94.

11. Furman, R.E., and Barchi, R.L. 1978. The pathophysiology of myotonia produced by aromatic carboxylic acids. Ann. Neurol. 4:357-365.

12. Franke, C., Hatt, H., Iaizzo, P.A., and LehmannHorn, F. 1990. Characteristics of $\mathrm{Na}^{+}$channels and
$\mathrm{Cl}$ - conductance in resealed muscle fibre segments from patients with myotonic dystrophy. J. Physiol. 425:391-405.

13. Renaud, J.F., et al. 1986. Expression of apamin receptor in muscles of patients with myotonic muscular dystrophy. Nature. 319:678-680.

14. Behrens, M.I., Jalil, P., Serani, A., Vergara, F., and Alvarez, O. 1994. Possible role of apamin-sensitive $\mathrm{K}+$ channels in myotonic dystrophy. Muscle Nerve. 17:1264-1270

15. Ebralidze, A., Wang, Y., Petkova, V., Ebralidse, K., and Junghans, R.P. 2004. RNA leaching of transcription factors disrupts transcription in myotonic dystrophy. Science. 303:383-387.

16. Mercatante, D.R., Sazani, P., and Kole, R. 2001 Modification of alternative splicing by antisense oligonucleotides as a potential chemotherapy for cancer and other diseases. Curr. Cancer Drug Targets. 1:211-230.

17. Lueck, J.D., et al. 2007. Chloride channelopathy in myotonic dystrophy resulting from loss of posttranscriptional regulation for CLCN1. Am. J. Physiol. Cell Pbysiol. 292:C1291-C1297.

18. Benders, A.A., Groenen, P.J., Oerlemans, F.T., Veerkamp, J.H., and Wieringa, B. 1997. Myotonic dystrophy protein kinase is involved in the modulation of the $\mathrm{Ca} 2+$ homeostasis in skeletal muscle cells. J. Clin. Invest. 100:1440-1447.

19. Benders, A.A., Wevers, R.A., and Veerkamp, J.H. 1996. Ion transport in human skeletal muscle cells: disturbances in myotonic dystrophy and Brody's disease. Acta. Physiol. Scand. 156:355-367.

20. Kimura, T., et al. 2005. Altered mRNA splicing of the skeletal muscle ryanodine receptor and sarcoplasmic/endoplasmic reticulum Ca2+-ATPase in myotonic dystrophy type 1. Hum. Mol. Genet. 14:2189-2200.

21. Dominski, Z., and Kole, R. 1993. Restoration of correct splicing in thalassemic pre-mRNA by antisense oligonucleotides. Proc. Natl. Acad. Sci. U. S. A. 90:8673-8677.
22. Dunckley, M.G., Manoharan, M., Villiet, P., Eperon, I.C., and Dickson, G. 1998. Modification of splicing in the dystrophin gene in cultured $\mathrm{Mdx}$ muscle cells by antisense oligoribonucleotides. Hum. Mol. Genet. 7:1083-1090.

23. Wilton, S.D., et al. 1999. Specific removal of the nonsense mutation from the $\mathrm{mdx}$ dystrophin mRNA using antisense oligonucleotides. Neuromuscul. Disord. 9:330-338.

24. Alter, J., et al. 2006. Systemic delivery of morpholino oligonucleotide restores dystrophin expression bodywide and improves dystrophic pathology. Nat. Med. 12:175-177.

25. Trip, J., Drost, G., van Engelen, B.G., and Faber, C.G. 2006. Drug treatment for myotonia. Cochrane Database Syst. Rev. CD004762.

26. Mankodi, A., et al. 2000. Myotonic dystrophy in transgenic mice expressing an expanded CUG repeat. Science. 289:1769-1773.

27. McMahon, J.M., Signori, E., Wells, K.E., Fazio, V.M., and Wells, D.J. 2001. Optimisation of electrotransfer of plasmid into skeletal muscle by pretreatment with hyaluronidase - increased expression with reduced muscle damage. Gene Ther. 8:1264-1270.

28. Parsons, S.A., Wilkins, B.J., Bueno, O.F., and Molkentin, J.D. 2003. Altered skeletal muscle phenotypes in calcineurin Aalpha and Abeta gene-targeted mice. Mol. Cell. Biol. 23:4331-4343.

29. Kanadia, R.N., et al. 2006. Reversal of RNA missplicing and myotonia after muscleblind overexpression in a mouse poly(CUG) model for myotonic dystrophy. Proc. Natl. Acad. Sci. U. S. A. 103:11748-11753.

30. Difranco, M., Neco, P., Capote, J., Meera, P., and Vergara, J.L. 2006. Quantitative evaluation of mammalian skeletal muscle as a heterologous protein expression system. Protein Expr. Purif. 47:281-288.

31. Hamill, O.P., Marty, A., Neher, E., Sakmann, B., and Sigworth, F.J. 1981. Improved patch-clamp techniques for high-resolution current recording from cells and cell-free membrane patches. Pflugers Arch. 391:85-100. 